\author{
Military Technical College \\ Kobry El-Kobbah, \\ Cairo, Egypt
}

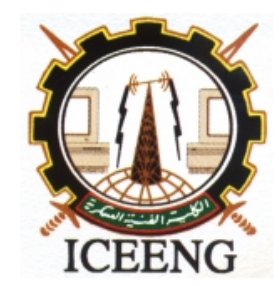

\title{
Analogy between Arithmetic Fuzzy Logic-based Representation Approach and Conventional Fuzzy Theory for Modeling and Optimization in Fully Fuzzy Environment
}

\author{
$7^{\text {th }}$ International Conference \\ on Electrical Engineering \\ ICEENG 2010
}

\author{
By \\ Walaa Ibrahim Mahmoud Gabr*
}

\section{$\underline{\text { Abstract: }}$}

This paper presents further development of the arithmetic fuzzy logic-based representation. The concept was originally proposed by Gabr and Dorrah for linear and nonlinear system using the notion of the normalized fuzzy matrices. The investigation is based on the dual cell representation, expressed by replacing each parameter with a pair of parentheses, the first is the actual value and the second is corresponding fuzzy level. The analogy of the proposed Arithmetic Fuzzy Logic-based Representation technique with the Conventional Fuzzy Theory are derived for various cases of operations of addition, subtraction, multiplication and division. It is shown that the suggested approach is identical to that of the conventional fuzzy theory for addition operations and gives average weighted fuzziness interval results of the subtraction operations. Moreover, it yields similar results of multiplications and divisions operations after ignoring the second order relative variations terms. Finally, the suggested approach is demonstrated to offer additional advantages of linearity, reversibility, simplicity, and applicability.

\section{Keywords:}

Conventional Fuzzy Theory, Fuzzy-based Logic Algebra, Normalized Fuzzy Matrices, Arithmetic Fuzzy Logic-based Representation

* Egyptian Holding Company of Electricity, Ministry of Electricity and Energy, Abbassia Square, Cairo, Egypt (e-mail: walaa_gabr@yahoo.com) 


\section{Introduction:}

Handling uncertainties in system modeling and optimization has been addressed heavily in the literature [1]-[4]. The techniques range for their complexity from simple sensitivity analysis, to more sophisticated stochastic modeling and end at the fuzzy modeling. The fuzzy modeling technique in the other hand implements the conventional fuzzy theory or fuzzy matrices. The concept of fuzzy matrices was elaborated by many researchers. Fuzzy matrices are another type of fuzzy representations. Different types of these matrices are defined as Fuzzy Matrices, Fuzzy BiMatrices, Fuzzy Super Matrices, and Fuzzy Interval Matrices [5]. The various types of fuzzy matrices are in fact inter-related.

In the analysis of Gabr and Dorrah [1]-[4], every parameter uncertainty is modeled using the proposed arithmetic fuzzy logic-based representation based on the normalized fuzzy matrices. The approach is based simply on the assignment of corresponding fuzzy levels for parameters uncertainty that is made in a heuristic way circumventing the previous difficulty in assuming probabilistic or membership functions. The approach involves only arithmetic (with no logic) operations for fuzzy processing.

In the present work, the Arithmetic Fuzzy Logic-based Representation defined on the Normalized Fuzzy Matrices is further elaborated. The analogy of the approach with the Conventional Fuzzy Theory [6]-[7] is derived, demonstrating the key feature of the suggested Arithmetic Fuzzy Logic-based Representation approach.

\section{Brief Description of Arithmetic Fuzzy Logic-based Representation}

The normalized fuzzy logic-based is based on representing each parameter $x$ by two components: $x_{0}$ the deterministic equivalence, and $X_{f}$ the fuzzy equivalence representing a small uncertainty or value tolerance in the parameter $x$ [1]-[4]. The term $X_{f}$ is modeled by the formula: $X_{f}=f_{r} \lambda_{x} X_{o}$ where $f_{r}$ is the relative unit fuzziness (usually a certain small percentage), and $\lambda_{x}$ is the corresponding fuzzy level. For the sake of simplicity $f_{r}$ is omitted in the representation and the parameter $x$ is expressed by the following pair:

$$
X=\left(X_{o}, \lambda_{x}\right)
$$

where the first term in the pair is the equivalent deterministic component, and $\lambda_{x}$ is an integer value indicating the corresponding fuzzy level of $X$. The scaled or normalized fuzzy term is $\tilde{X}=f_{x} \cdot \lambda_{x}$, such that $|\tilde{X}|<1$. In general, the fuzzy level could be extended from level $-p$ to $+m$ where $p$ and $m$ are positive integers. In this case, the 
value of the relative unit fuzziness $f_{r}$ is restricted such as $p . f_{r}<1$ and $m . f_{r}<1$ (preferably will be $<<1$ ). This will guarantee not changing the sign of the parameters during the formulation, and that all the scaled or normalized fuzzy components satisfy the conditions of the normalized fuzzy matrices boundary $[-1,+1]$. This is not a real restriction as the whole fuzzy process is mainly based on intuitive estimation and heuristic evaluations. Similarly, we have for any other two general parameters $Y$ and $Z$ the following representations:

$$
Y=\left(Y_{o}, \lambda_{y}\right)
$$

and

$$
Z=\left(Z_{o}, \lambda_{z}\right) \text {. }
$$

The algebra of Arithmetic fuzzy logic-based representation is developed for the basic operations as shown in Table 1 [1]-[4].

\section{Table 1}

Summary of Common Arithmetic Fuzzy Logic-Based Representation Algebra.

\begin{tabular}{|c|l|c|c|}
\hline Ser. & \multicolumn{1}{|c|}{$\begin{array}{c}\text { Name of } \\
\text { Operation }\end{array}$} & $\begin{array}{c}\text { Symbolic } \\
\text { Representation } \\
\text { of Operation }\end{array}$ & $\begin{array}{c}\text { Resulting Values and Fuzzy Levels } \\
\text { from Operation }\end{array}$ \\
\hline 1 & Multiplication & $\mathrm{X} . \mathrm{Y}$ & $X \mathbf{X}\left(X_{o} Y_{o}, \lambda_{x}+\lambda_{y}\right)$. \\
\hline 2 & Division & $\mathrm{X} / \mathrm{Y}$ & $X / Y=\left(X_{o} / Y_{o}, \lambda_{x}-\lambda_{y}\right)$ \\
\hline 3 & Addition & $\mathrm{X}+\mathrm{Y}$ & $Z_{o}=X_{o}+Y_{o}$, and $\lambda_{z}=\frac{\lambda_{x} X_{o}+\lambda_{y} Y_{o}}{X_{o}+Y_{o}}$ \\
\hline 4 & Subtraction & $\mathrm{X}-\mathrm{Y}$ & $Z_{o}=X_{o}-Y_{o}$, and $\lambda_{z}=\frac{\lambda_{x} X_{o}-\lambda_{y} Y_{o}}{X_{o}-Y_{o}}$ \\
\hline
\end{tabular}

For the sake of uncertainty analysis, it is assumed for each fuzzy level as an example that the parameter variation can be quantitatively modeled by a Gaussian probability function of zero mean and standard deviation $\sigma_{\lambda}$. In general, assumption of normality can be substantiated by applying the Central Limit theorem, which states that the addition of independent several probabilistic parameters will result in an overall normal distribution.

\section{Analogy between the Algebra of Conventional Fuzzy Theory and Arithmetic Fuzzy Logic-based Representation Approach}

\subsection{Basic Definitions}

We can uniquely describe two fuzzy number $A$ and $B$ as two collection of intervals i.e., 
$\left[a_{1}^{(\alpha)}, a_{2}^{(\alpha)}\right]$ and $\left[b_{1}^{(\alpha)}, b_{2}^{(\alpha)}\right]$ respectively. We recall that the $\alpha$-cuts of $A$ and $B$ were defined as the crisp sets

$$
A_{\alpha}=\left\{x \mid \mu_{A}(x) \geq \alpha\right\}
$$

and

$$
B_{\alpha}=\left\{x \mid \mu_{B}(x) \geq \alpha\right\}
$$

The $\alpha$-cuts in equations (4) and (5) are simply intervals on the $x$ axis, and hence for each $\alpha$ we have as shown in Figure 1 [7]-[8]:

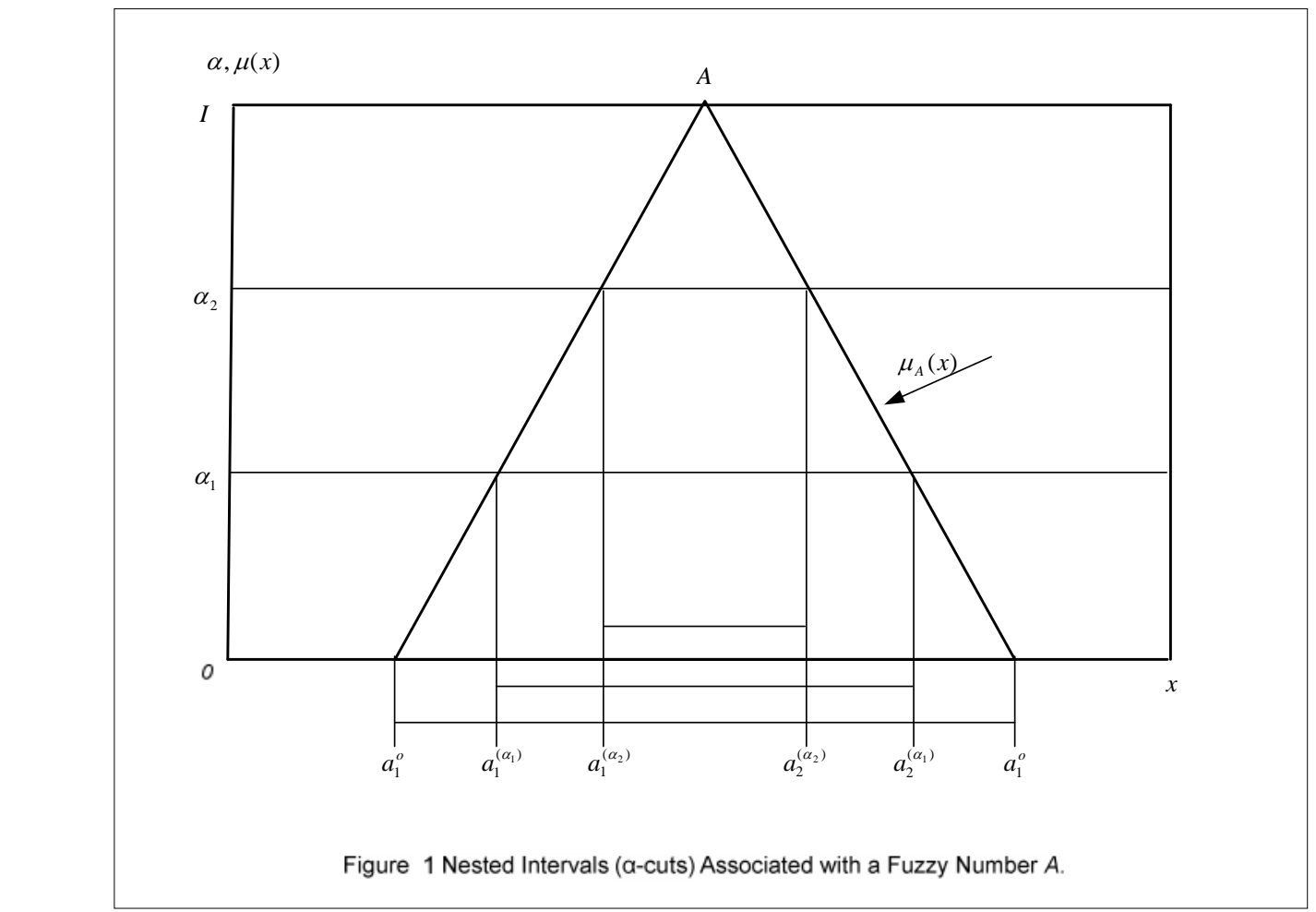

$$
A_{\alpha}=\left[a_{1}^{(\alpha)}, a_{2}^{(\alpha)}\right]
$$

and

$$
B_{\alpha}=\left[b_{1}^{(\alpha)}, b_{2}^{(\alpha)}\right] .
$$

The equivalence of the arithmetic fuzzy logic-based representation is as follows:

$$
A=\left(A_{o}, \lambda_{A}\right)
$$

and

$$
B=\left(B_{o}, \lambda_{B}\right)
$$

where assuming an arbitrary fixed single end, such that 


$$
\begin{aligned}
& A_{o}=a_{1}^{(\alpha)} \\
& \lambda_{A}=\frac{\left(a_{2}^{(\alpha)}-a_{1}^{(\alpha)}\right)}{A_{o} \cdot f_{r}} \\
& B_{o}=b_{1}^{(\alpha)}
\end{aligned}
$$

and

$$
\lambda_{B}=\frac{\left(b_{2}^{(\alpha)}-b_{1}^{(\alpha)}\right)}{B_{o} \cdot f_{r}}
$$

where $f_{r}$ indicates the relative fuzziness such that $f_{r}<1$ or preferably $f_{r} \ll 1$. The fuzzy level is normally rounded off to line nearest integer value. It is assumed in the analysis of the fuzzy level representation that necessary the second order relative variations are ignored, that is for $\Delta A=\left(a_{2}^{(\alpha)}-a_{1}^{(\alpha)}\right)$ and $\Delta B=\left(b_{2}^{(\alpha)}-b_{1}^{(\alpha)}\right)$, then $\frac{\Delta A}{A_{0}} \cdot \frac{\Delta A}{A_{0}}$, $\frac{\Delta B}{B_{0}} \cdot \frac{\Delta B}{B_{0}}$ and $\frac{\Delta A}{A_{0}} \cdot \frac{\Delta B}{B_{0}}$ are very small and can be ignored.

\subsection{Analogy of the Addition Operation}

The addition of $A$ and $B$ may be defined in terms of addition of the $\alpha$-cuts of the two numbers as follows:

$$
A+B \equiv\left[a_{1}^{(\alpha)}, a_{2}^{(\alpha)}\right]+\left[b_{1}^{(\alpha)}, b_{2}^{(\alpha)}\right]
$$

where $\left[a_{1}^{(\alpha)}, a_{2}^{(\alpha)}\right]$ is the collection of intervals representing the fuzzy number $A$, and $\left[b_{1}^{(\alpha)}, b_{2}^{(\alpha)}\right]$ is the collection of intervals representing the fuzzy number $B$. Intervals are added by adding their corresponding left and right endpoints, and therefore (14) becomes

$$
A+B=\left[a_{1}^{(\alpha)}+b_{1}^{(\alpha)}, a_{2}^{(\alpha)}+b_{2}^{(\alpha)}\right]
$$

Now, if $x$ and $y$ are variables, obviously their sum may be thought of as a function of $x$ and $y$; that is,

$$
z(x, y)=x+y
$$

According to the extension principle, their sum is a fuzzy set on $z$ denoted as $C$, whose membership function is

$$
\mu_{C}(z) \equiv \underset{z=x+y}{\vee}\left[\mu_{A}(x) \wedge \mu_{B}(y)\right]
$$


In the corresponding arithmetic fuzzy logic-based representation we have

$$
C=A+B=\left(C_{o}, \lambda_{c}\right)
$$

where

$$
C_{o}=A_{o}+B_{o}
$$

and

$$
\lambda_{c}=\frac{\lambda_{A} \cdot A_{o}+\lambda_{B} \cdot B_{o}}{A_{o}+B_{o}}
$$

The corresponding arithmetic fuzzy logic-based representation, we have after substituting (11) and (13) into (20), the following

$$
\begin{aligned}
\lambda_{c} & =\frac{\lambda_{A} \cdot A_{o}+\lambda_{B} \cdot B_{o}}{A_{o}+B_{o}} \\
& =\frac{\left(a_{2}^{(\alpha)}+b_{2}^{(\alpha)}-a_{1}^{(\alpha)}-b_{1}^{(\alpha)}\right)}{C_{o} \cdot f_{r}}
\end{aligned}
$$

which gives the same interval described in (15).

\subsection{Analogy of the Subtraction Operation}

The difference $C$ of two fuzzy numbers $A, B$ may be defined either through interval subtraction utilizing the $\alpha$-cut representation of the two numbers or through the extension principle. Using $\alpha$-cuts we subtract them as follows

$$
A-B \equiv\left[a_{1}^{(\alpha)}, a_{2}^{(\alpha)}\right]-\left[b_{1}^{(\alpha)}, b_{2}^{(\alpha)}\right]
$$

where $\left[a_{1}^{(\alpha)}, a_{2}^{(\alpha)}\right]$ is the collection of closed intervals representing $A$, and $\left[b_{1}^{(\alpha)}, b_{2}^{(\alpha)}\right]$ is the collection of closed intervals representing $B$. Two intervals are subtracted by subtracting their left and right endpoints, and thus (22) becomes

$$
A-B=\left[a_{1}^{(\alpha)}-b_{2}^{(\alpha)}, a_{2}^{(\alpha)}-b_{1}^{(\alpha)}\right]
$$

The membership function of $C=A-B$ can be computed from

$$
\mu_{A-B}(z) \equiv \underset{z=x-y}{\vee}\left[\mu_{A}(x) \wedge \mu_{B}(y)\right]
$$

In the corresponding arithmetic fuzzy logic-based representation, we have

$$
C=A-B=\left(C_{o}, \lambda_{c}\right)
$$

where

$$
C_{o}=A_{o}-B_{o}
$$


and

$$
\lambda_{c}=\frac{\lambda_{A} \cdot A_{o}-\lambda_{B} \cdot B_{o}}{A_{o}-B_{o}}
$$

The corresponding arithmetic fuzzy logic-based representation, we have after substituting of (11) to (13) into (27), we get the following:

$$
\begin{aligned}
\lambda_{c} & =\frac{\lambda_{A} \cdot A_{o}-\lambda_{B} \cdot B_{o}}{A_{o}-B_{o}} \\
& =\frac{\left(a_{2}^{(\alpha)}-b_{1}^{(\alpha)}\right)-\left(a_{1}^{(\alpha)}-b_{2}^{(\alpha)}\right)}{\left(A_{o}-B_{o}\right) \cdot f_{r}}
\end{aligned}
$$

which gives the average weighted fuzziness results of the interval as an approximation of interval described in (23).

\subsection{Analogy of the Multiplication Operation}

In conventional fuzzy theory, the fuzzy number multiplication may be defined either as $\alpha$-cut multiplication or through the extension principle. Using the $\alpha$-cut representation of two numbers $A, B$ their product is defined as

$$
A \cdot B=\left[a_{1}^{(\alpha)}, a_{2}^{(\alpha)}\right] \cdot\left[b_{1}^{(\alpha)}, b_{2}^{(\alpha)}\right] .
$$

In general, the product of two intervals is a new interval equivalent to:

$$
A \cdot B=\left[\begin{array}{cc}
a_{1}^{(\alpha)} \cdot b_{1}^{(\alpha)}, a_{2}^{(\alpha)} \cdot b_{2}^{(\alpha)} \\
1
\end{array}\right] .
$$

The extension principle indicates that their product is a fuzzy set on $z$, denoted as $A . B$, whose membership function is

$$
\mu_{A . B}(z) \equiv \underset{z=x . y}{\vee}\left[\mu_{A}(x) \wedge \mu_{B}(y)\right]
$$

In the corresponding arithmetic fuzzy logic-based representation we have

$$
C=A \cdot B=\left(C_{o}, \lambda_{c}\right) .
$$

where

$$
C_{o}=A_{o} \cdot B_{o}
$$

and

$$
\lambda_{c}=\lambda_{A}+\lambda_{B} .
$$

Now, for the fuzzy level $\lambda_{c}$, we apply (11) and (13) into (34) to give after neglecting second order relative variation terms:

$$
\lambda_{c}=\frac{\left(a_{2}^{(\alpha)}-a_{1}^{(\alpha)}\right)}{A_{o} \cdot f_{r}}+\frac{\left(b_{2}^{(\alpha)}-b_{1}^{(\alpha)}\right)}{B_{o} \cdot f_{r}}
$$




$$
=\frac{\left(b_{2}^{(\alpha)} a_{2}^{(\alpha)}-a_{1}^{(\alpha)} b_{1}^{(\alpha)}\right)}{\left(A_{o} \cdot B_{o}\right) \cdot f_{r}}
$$

this gives the interval $\left(b_{2}^{(\alpha)} a_{2}^{(\alpha)}-a_{1}^{(\alpha)} b_{1}^{(\alpha)}\right)$ which is similar to the conventional theory shown in (30).

\subsection{Analogy of the Division Operation}

We can find the quotient of two fuzzy numbers $A$ and $B$ either through interval division or by the extension principle. In terms of their $\alpha$-cut representation, we write the quotient of the two numbers as

$$
A / B=\left[a_{1}^{(\alpha)}, a_{2}^{(\alpha)}\right] /\left[b_{1}^{(\alpha)}, b_{2}^{(\alpha)}\right]
$$

In general, the quotient of two intervals is a new interval given by

$$
\left[a_{1}^{(\alpha)}, a_{2}^{(\alpha)}\right] /\left[b_{1}^{(\alpha)}, b_{2}^{(\alpha)}\right] \equiv\left[\begin{array}{ll}
a_{1}^{(\alpha)} & \frac{a_{2}^{(\alpha)}}{b_{2}^{(\alpha)}} \\
b_{1}^{(\alpha)}
\end{array}\right]
$$

Hence, provided that $b_{2}^{(\alpha)} \neq 0$ and $b_{1}^{(\alpha)} \neq 0$, the quotient of $A, B$ is

$$
A / B=\left[\begin{array}{ll}
a_{1}^{(\alpha)} \\
b_{2}^{(\alpha)} & \frac{a_{2}^{(\alpha)}}{b_{1}^{(\alpha)}}
\end{array}\right]
$$

The extension principle indicates that $A / B$ is a fuzzy set with membership function

$$
\mu_{A / B}(z) \equiv \underset{z=x, y}{\vee}\left[\mu_{A}(x) \wedge \mu_{B}(y)\right]
$$

Similarly for the corresponding arithmetic fuzzy logic-based representation, we have

$$
C=A / B=\left(C_{o}, \lambda_{c}\right)
$$

where

$$
C_{o}=A_{o} / B_{o}
$$

and

$$
\lambda_{c}=\lambda_{A}-\lambda_{B}
$$

Now, for the fuzzy level $\lambda_{c}$, we apply (11), (13) into (42). This gives after neglecting the second order relative variation terms:

$$
\begin{aligned}
\lambda_{c} & =\frac{\left(a_{2}^{(\alpha)}-a_{1}^{(\alpha)}\right)}{A_{o} \cdot f_{r}}-\frac{\left(b_{2}^{(\alpha)}-b_{1}^{(\alpha)}\right)}{B_{o} \cdot f_{r}} \\
& =\frac{B_{o}}{A_{o} \cdot f_{r}}\left(\frac{a_{2}^{(\alpha)}}{b_{1}^{(\alpha)}}-\frac{a_{1}^{(\alpha)}}{b_{2}^{(\alpha)}}\right)
\end{aligned}
$$


which is similar to the result of (38) given by the conventional fuzzy logic concept.

\section{Summary of Results:}

The above analysis results are summarized in Table 2.

Table 2 Analogy between the Algebra of Conventional Fuzzy Theory and Arithmetic Fuzzy Logic-based Representation Approach.

\begin{tabular}{|c|c|c|}
\hline $\begin{array}{l}\text { Algebraic } \\
\text { Operation }\end{array}$ & Conventional Fuzzy Theory & $\begin{array}{l}\text { Suggested Arithmetic Fuzzy } \\
\text { Logic-based Representation }\end{array}$ \\
\hline$C=A+B$ & $\begin{array}{l}A+B=\left[a_{1}^{(\alpha)}+b_{1}^{(\alpha)}, a_{2}^{(\alpha)}+b_{2}^{(\alpha)}\right] \\
\text { Or equivalently for } \\
\mu_{C}(z) \equiv \underset{z=x+v}{\vee}\left[\mu_{A}(x) \wedge \mu_{B}(y)\right]\end{array}$ & $\begin{array}{l}C_{o}=A_{o}+B_{o} \text { and } \\
\lambda_{c}=\frac{\lambda_{A} \cdot A_{o}+\lambda_{B} \cdot B_{o}}{A_{o}+B_{o}}\end{array}$ \\
\hline$C=A-B$ & $\begin{array}{l}A-B=\left[a_{1}^{(\alpha)}-b_{2}^{(\alpha)}, a_{2}^{(\alpha)}-b_{1}^{(\alpha)}\right] \\
\text { Or equivalently for } \\
\mu_{A-B}(z) \equiv \underset{z=x-y}{\vee}\left[\mu_{A}(x) \wedge \mu_{B}(y)\right]\end{array}$ & $\begin{array}{l}C_{o}=A_{o}-B_{o} \text { and } \\
\lambda_{c}=\frac{\lambda_{A} \cdot A_{o}-\lambda_{B} \cdot B_{o}}{A_{o}+B_{o}}\end{array}$ \\
\hline$C=A \cdot B$ & $\begin{array}{l}A \cdot B=\left[a_{1}^{(\alpha)} \cdot b_{1}^{(\alpha)}, a_{2}^{(\alpha)} \cdot b_{2}^{(\alpha)}\right] \\
\text { Or equivalently for } \\
\mu_{A . B}(z) \equiv \underset{z=x . y}{\vee}\left[\mu_{A}(x) \wedge \mu_{B}(y)\right]\end{array}$ & $C_{o}=A_{o} \cdot B_{o}$ and $\lambda_{c}=\lambda_{A}+\lambda_{B}$ \\
\hline$C=A / B$ & $\begin{array}{l}A / B=\left[\frac{a_{1}^{(\alpha)}}{b_{2}^{(\alpha)}}, \frac{a_{2}^{(\alpha)}}{b_{1}^{(\alpha)}}\right] \\
\text { Or equivalently for } \\
\mu_{A / B}(z) \equiv \underset{z=x, y}{\vee}\left[\mu_{A}(x) \wedge \mu_{B}(y)\right]\end{array}$ & $C_{o}=A_{o} / B_{o}$ and $\lambda_{c}=\lambda_{A}-\lambda_{B}$ \\
\hline
\end{tabular}

$* \vee($.$) means maximum of (.), while \wedge$ (.) indicates minimum of (.).

\section{Conclusions:}

The new arithmetic fuzzy logic-based representation proposed based on Normalized Fuzzy Matrices and originally developed by Gabr and Dorrah [1]-[4] is further developed in this paper. It is shown that the suggested approach is identical to that of the conventional fuzzy theory for addition operations and gives average weighted fuzziness interval results of the subtraction operations. Moreover, it yields similar results of multiplications and divisions operations after ignoring the second order relative variations terms. 
It appears from the above investigation that the proposed arithmetic fuzzy logic-based representation has the same mathematical function of the conventional fuzzy theory. However, the new approach provides a much easier calculations forum that makes its application much practical and effective. Moreover, it follows from the above mathematical derivations that the Arithmetic Fuzzy Logic-based Representation approach possesses the following key features over the conventional fuzzy theory, namely: Linearity, Reversibility, Simplicity and Applicability. The approach is pragmatic as it requires only specifying heuristically the fuzzy logic-based levels of the parameters and coefficients, which can be relatively evaluated in real life. These levels are then transferred at the end of solution to actual uncertainties.

\section{Acknowledgements}

The author would like to thank Dr. Hassen T. Dorrah, Professor of Electrical Engineering, Cairo University, Egypt, for his valuable comments and suggestions.

\section{References:}

[1] Walaa Ibrahim Gabr and Hassen Taher Dorrah, "New Fuzzy Logic-based Arithmetic and Visual Representations for Systems' Modeling and Optimization" IEEE International Conference on Robotics and Biomimetics, Bangkok, Thailand, 22-25 February 2009, pp. 715-722.

[2] Walaa Ibrahim Gabr and Hassen Taher Dorrah, "Development of Fuzzy Logicbased Arithmetic and Visual Representations for Systems' Modeling and Optimization of Interconnected Networks", IEEE International Conference on Robotics and Biomimetics, Bangkok, Thailand, 22-25 February 2009, pp. 723-730.

[3] Hassen Taher Dorrah and Walaa Ibrahim Gabr, "Multi-Objective Linear Optimization Using Fuzzy Logic-based Arithmetic and Visual Representations with Forward and Backward Tracking ", IEEE International Conference on Robotics and Biomimetics, Bangkok, Thailand, 22-25 February 2009, pp. 731-738.

[4] Hassen Taher Dorrah and Walaa Ibrahim Gabr, "Development of Fuzzy Logicbased Arithmetic and Visual Representations for Solving Quadratic Programming in Fully Fuzzy Environment" IEEE International Conference on Information and Automation, Zhuhai, China, 22-25 June, 2009, pp. 46-53.

[5] W. B. Vasantha Kandasamy and Florentin Smarandache, Fuzzy Interval Matrices, Neutrosophic Interval Matrices and Their Applications, URL: http://www.gallup. nm.edu/ smarandache/NIM.pdf, HEXIS, Phoenix, Arizona, 2006.

[6] Lefteri H. Tsaukalcs, and Robert E. Uhrig, Fuzzy and Neural Approaching in Engineering, John Wiley \& Sons, Inc. New York, 1997.

[7] Kevin M. Passino and Stephen Yukovich, Fuzzy Control, Addison-Wesley Longman, Inc., Menlo Park, California, USA, 1998. 